\title{
Novel Evaluation of Digital Halftone Image Qualities by Psychological Analysis
}

\author{
Tadahiko Kimoto ${ }^{1}$, Chieko Kato ${ }^{2}$ \\ ${ }^{1}$ Faculty of Science and Engineering, Toyo University, Kawagoe, Japan; \\ ${ }^{2}$ Faculty of Information Sciences and Arts, Toyo University, Kawagoe, Japan; \\ ${ }^{1}$ kimoto@toyo.jp, ${ }^{2}$ kato-c@toyo.jp
}

\begin{abstract}
Monochrome binary halftone images express multiple levels with halftones produced by distributing black and white pixels. In this paper, for the subjective evaluation of halftone qualities, binary halftone images are considered as pointillistic images of visible black-and-white pixels. Visual impressions given by the pixel distribution are measured by the semantic differential (SD) method in a psychological manner.

An experiment for measuring subjective impressions of subjects by using selected 30 SD items was conducted on several binary halftone images that were produced by three kinds of the typical halftoning methods: two error-diffusion methods and a kind of random dither method. The sample data are analyzed statistically by factor analysis. As a result, two common factors are found out and interpreted as the sensitivity factor and the activity factor, respectively. Also, the result of variance analysis demonstrates that the two common factors are able to describe the difference among the three halftoning methods.
\end{abstract}

Keywords: Digital halftone, Visual impression, Semantic differential method, Factor analysis, Variance analysis.

\section{INTRODUCTION}

Digital halftoning is one of data compression techniques for displaying digital images. It reduces pixel bits while compensating the missing signal levels with visually pseudo-continuous levels, called halftones [1]. Halftoning to convert multi-level images to bi-level ones is important particularly in printing today. There are a variety of halftoning methods for the binarization [2].

In generating a monochrome binary halftone image, halftoning is achieved by distributing black and white pixels. As a result, the resulting image looks like a picture made in a pointillistic manner, which is a style of painting for making up pictures by using small colored dots. Appearances of the halftone image, which are essentially subjective qualities, are generally 
assessed by observing it at an image resolution such that each pixel is hardly distinguishable. However, it is just the pixel distribution that characterizes the halftones.

In this paper, we consider binary halftone images as the pointillistic images composed of visible black and white pixels rather than the images approximated with halftone levels. The purpose is to characterize halftoning methods from the viewpoint of visual impressions given by the black-and-white distribution. To measure the subjective impressions, we have carried out an experiment using a semantic differential (SD) method, which is often used for psychological analysis [3]. The sample data are investigated by statistical analysis to search for significant factors that can describe the halftone qualities.

\section{VARIETY OF BINARY HALFTONES}

\subsection{Principle of halftoning in binary}

The principle of converting a multi-level image to a bi-level or binary halftone image is expressed below: Let $f(m, n)$ denote a pixel value at the coordinates of $(m, n)$ in an image $f$. Also, source images are assumed to have 8-bit, that is, 256 levels from 0 (black) to 255 (white). Converting a source image $f$ to a binary image $f^{\prime}$ is generally expressed as

$$
f^{\prime}(m, n)= \begin{cases}1, & f(m, n)+a(m, n) \geq 128 \\ 0, & f(m, n)+a(m, n)<128\end{cases}
$$

where $a(m, n)$ is a signal that determines a threshold level for $f(m, n)$ as a result. Varying $a(m, n)$ for every pixel produces the variation of $f^{\prime}(m, n)$. Accordingly, the properties of the quantization error $e(m, n)$, which is defined by

$$
e(m, n)=255 f^{\prime}(m, n)-f(m, n)
$$

depend on the properties of $a(m, n)$. Different properties of the quantization error yield different distribution of black and white pixels, and accordingly, different appearances in pointillism.

\subsection{Halftoning methods being focused}

In this paper, we consider the following three typical ones of the conventional halftoning methods.

\subsubsection{Error diffusion halftoning}

The principle of error diffusion halftoning is to diffuse the quantization errors by transforming them to high-frequency signals that could be hardly perceptible to human eyes. The transformation is implemented with a digital filter, which gives the values of $a(m, n)$ of Equation (1). Thus, the frequency response characteristics of the filter determine the distribution of binary pixels and consequently, affect the halftone qualities. 
Various digital filters for error-diffusion halftoning have been known that are capable of producing halftones of visual quality. We have chosen two such filters in this paper: the filter proposed by Floyd and Steinberg [4] and that by Jarvis et al. [5]. We refer to the error-diffusion methods with these two filters as EDF-FS and EDF-JJN, respectively. According to the filter characteristics, EDF-FS diffuses the quantization errors in the high-frequency range, and EDFJJN does in the middle-frequency range. This reflects the difference in halftone quality for the two methods.

\subsubsection{Improved grayscale quantization}

Improved grayscale (IGS) quantization [6] performs halftoning like a random dither method. In halftoning an 8-bit source image by IGS, pixel values are accumulated through a scanning path in the image until at a pixel, say, the coordinates $(m, n)$, and the seven low-order bits of the result are used as $a(m, n)$. Consequently, $a(m, n)$ behaves as a random variable with local characteristics of the image.

In the experiment below, IGS is carried out with a level transformation for preserving the average level of a source image through halftoning in terms of 8-bit levels [7]. Also, a Hilbert scanning path is used to implement IGS in a source image so that artifacts such as geometric patterns can be prevented from occurring in the resulting image. We refer to the whole scheme as HT-IGS below.

\section{EXPERIMENTAL METHOD}

\subsection{Preparing test images}

We use monochrome images of 8-bit brightness levels from 0 to 255 as the original images, and convert them to binary halftone images for the experiment. The procedure of the conversion is composed of the following three processes:

(1) Each original image is modified by the histogram equalization transformation [8] so that all source images to which the halftoning methods are applied can have both the same contrast and the same average level. The resulting image, $f$, has pixel levels ranging in the whole of 8-bit levels, and all the levels occur equally often.

(2) A binary image $f^{\prime}$ is generated from $f$ by each method of EDF-FS, EDF-JJN and HT-IGS.

(3) The pixel values 0 and 1 of $f^{\prime}$ are transformed to the darkest level 0 and the brightest level 255, respectively.

Thus, the source images are normalized in terms of overall contrast and averaged brightness level. Hence, the halftone patterns rather than the halftone levels are expected to mainly affect impressions of the test images. 


\subsection{Displaying test images}

We decide a method for presenting the test images to subjects by specifying image resolution and viewing conditions. The image resolution is related both to image size on a monitor screen and to a distance between the monitor and a viewer. The viewing conditions are involved in implementing the SD method.

\subsubsection{Image resolution}

We use a 17-inch, 24-bit-color TFT LCD monitor of 1,280 x 1,024 dots to display the test images with. The region of $512 \times 400$ pixels of a test image is shown in the middle of the screen by using two by two dots for every pixel. Thus, the displayed image has the size of about 270 $\mathrm{mm}$ in width and about $211 \mathrm{~mm}$ in height, in other words, the resolution of about 48 pixels per inch on the screen. A subject looks at the monitor about three times as long as the monitor's height away. The black and white pixels look generally distinguishable from the distance.

\subsubsection{Viewing conditions}

There are two conditions to consider for viewing the test images on a monitor screen.

(a) Displaying time; the duration of time a subject looks at a test image must be the same both for any subject and for any test image. It should be long enough for the subject to gain some visual impressions from the image but not so long as to make the subject tired of looking at it.

(b) Background color on the screen; a test image is displayed in the middle of the monitor screen. Hence, a color of the area surrounding the image, that is, the background influences the easiness in looking at the image.

To examine these conditions, we have conducted a preliminary experiment. The conditions have been determined from the result: The displaying time is set four seconds, and the background color is set black (See Appendix A).

\subsection{Semantic differential method}

An SD item is a pair of adjective words that are contradictory to each other, for example, fine-coarse. It should represent a visual impression of pictures in the present study. The SD items that seem to apply to the halftone images are determined in the following manner (See Appendix B for more details): First, a total of 75 items are chosen from among each of seven categories, which are generally used in psychological analysis. Then, we have carried out another preliminary experiment where subjects look at several halftone images and select those items that seem to describe visual impressions of the images. From the result, 30 of the most selected items have been determined for the use in this study. The items will be shown later (in Table 1).

As shown in Appendix B.2, for each of the selected items, a scale of seven gradations from 1 to 7 is defined to measure the impression that the item describes. For example, for the item 
dark-bright, the gradation of 1 represents dark, and 7 represents bright, while 4 represents not impressed either way (See Figure A.7). All the items each expressed by the corresponding scale are listed in a questionnaire sheet to present to subjects.

\subsection{Conducting the experiment}

\subsubsection{Test images used for SD method}

Figure 1 shows five source images from which the test halftone images are generated. We have selected these images considering the following points:

(a) The images hardly include anything such that some ones like it personally and some ones dislike it, such as some kinds of vegetables.

(b) There are included a variety of contents in the image set: a living thing, a nonliving thing, a plant, a human, a close-range view, a long-distant view and so on.

A total of 15 binary halftone images have been produced from these original images by using the three halftoning methods. Examples of the halftone image produced by each method are shown in Figure 2. These images are to be used only for the SD method, which have never been used in the preliminary experiments described in Appendices.

\subsubsection{Procedure of SD method}

Thirty-nine subjects, who are all Japanese in their early twenties and unfamiliar with halftoning techniques, have participated in the experiment. The 15 test images are displayed on the monitor one by one in a random order to a subject. The SD method proceeds for every subject as follows:

(1) The subject is asked to gaze at the image while it is being displayed on the monitor screen.

(2) As soon as the image is erased from the screen, the subject is asked to evaluate each SD item with the corresponding scale.

(3) After evaluating all the SD items, the subject goes on to the next image by operating the experimental system himself/herself.

The experiment has been carried out for every subject in the same indoor environment of, for example, the lighting. 

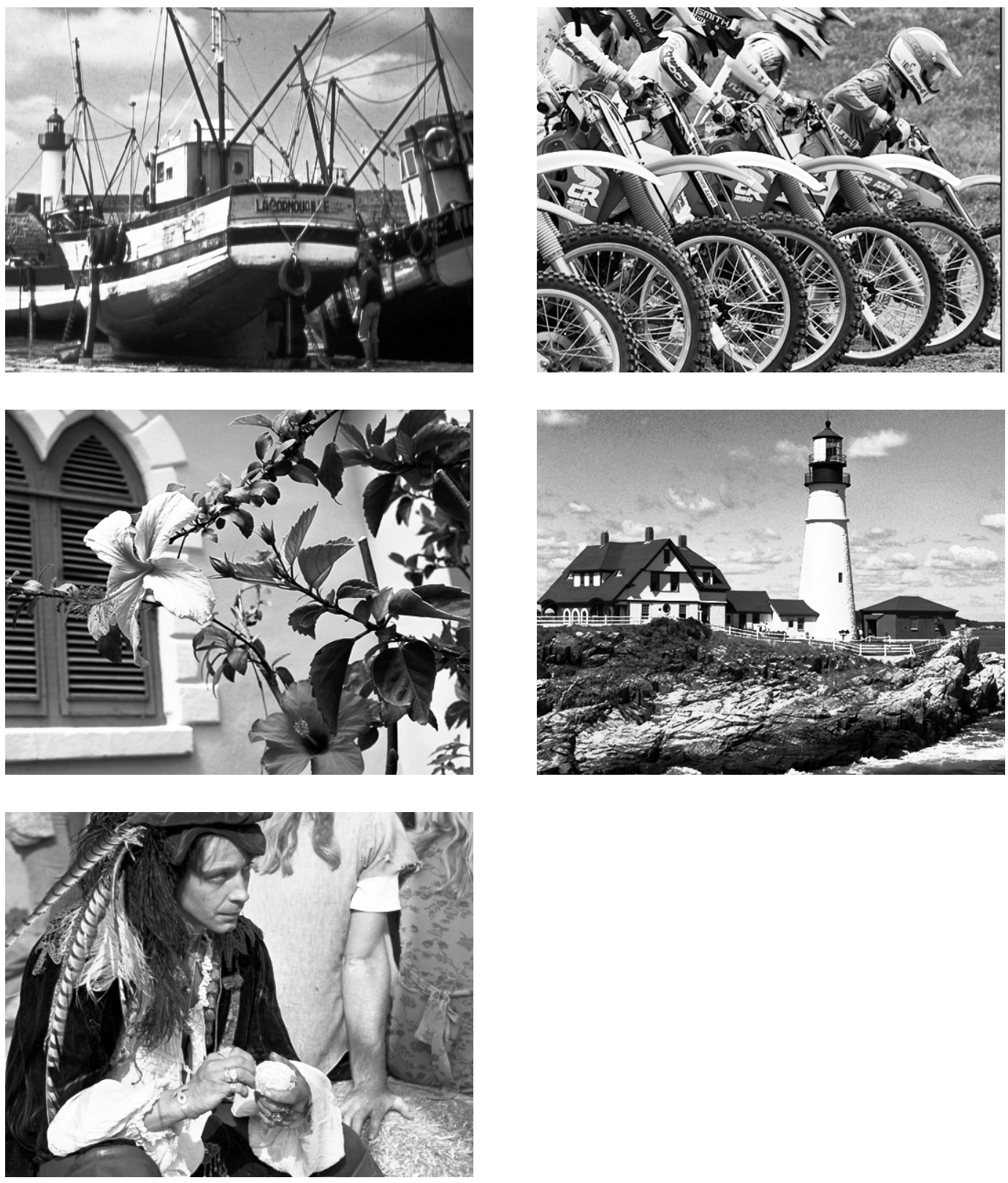

Figure 1: Monochrome 8-bit images used as source images to produce halftone images from, which are to be used in the main experiment of the SD method. Each figure shows the $512 \times 400$ region of the image that is actually displayed on the monitor while the whole image is of $512 \times 512$ pixels. These images have been processed in the manner described in Section 3.1. The original images are from the WEB sites [9-11]. 


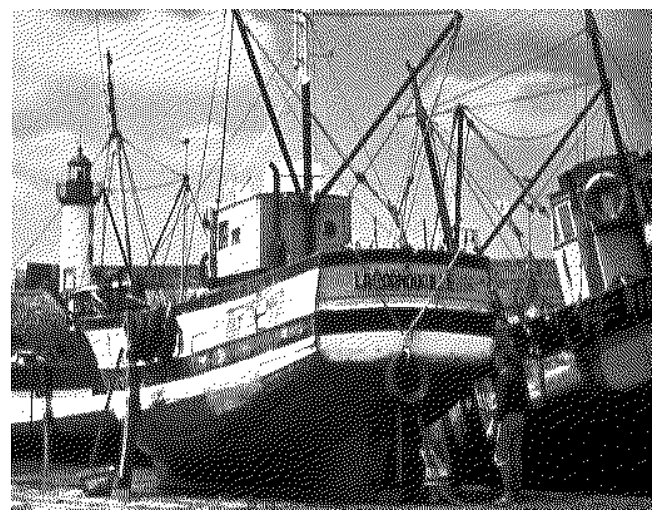

(a)

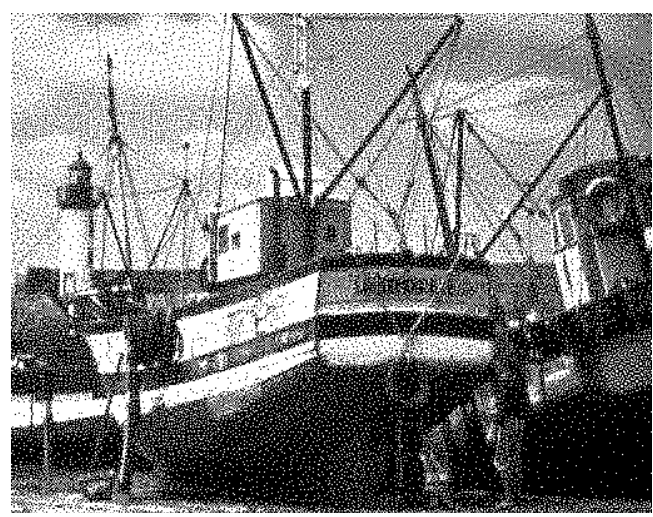

(c)

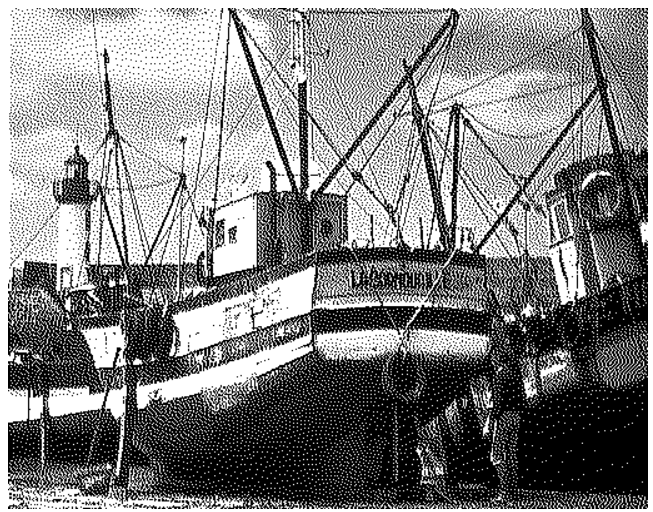

(b)

Figure 2: Examples of $512 \times 400$ halftone images, (a) by EDF-FS, (b) by EDF-JJN, and (c) by HT-IGS

\section{ANALYSES OF EXPERIMENTAL RESULT}

\subsection{Factor analysis}

\subsubsection{Deriving common factors}

Thirty-nine sample data of 30 SD-item values have been obtained from the experiment for each of five source images and for each of three methods. Considering each SD item as a variable that takes a value in $1,2, \ldots, 7$ of the item's scale, we carry out a factor analysis of the 30-variable data. In the analysis, the maximum likelihood method is used for the calculation of factors, and the axis rotation is carried out by the varimax rotation method. The analysis proceeds as follows.

First, all the 585 sample data are analyzed to derive common factors from the variables. The result has shown that there are five factors with the eigenvalue greater than one. Taking account of the contribution of the factors, we have set the number of common factors to be extracted to two, and then, carry out the factor analysis again to obtain them. 
Table 1: Result of factor analysis

\begin{tabular}{|c|c|c|c|}
\hline \multirow[b]{2}{*}{ No. } & \multirow{2}{*}{$\begin{array}{c}\text { SD item } \\
\text { word of value } 1 \text {-word of value } 7\end{array}$} & \multicolumn{2}{|c|}{ Factor loading } \\
\hline & & Factor 1 & Factor 2 \\
\hline 1 & unlikeable-likeable & 0.902 & 0.074 \\
\hline 2 & bad-good & 0.897 & 0.135 \\
\hline 3 & messy-tidy & 0.871 & 0.028 \\
\hline 4 & unfriendly-friendly & 0.863 & 0.019 \\
\hline 5 & ugly-beautiful & 0.844 & -0.045 \\
\hline 6 & low quality-high quality & 0.841 & 0.155 \\
\hline 7 & dislike-like & 0.828 & 0.096 \\
\hline 8 & unclear-clear & 0.792 & 0.237 \\
\hline 9 & dirty-clean & 0.770 & -0.178 \\
\hline 10 & unfocused-easy to recognize & 0.768 & 0.264 \\
\hline 11 & dull-vivid & 0.767 & 0.072 \\
\hline 12 & unnatural-natural & 0.757 & -0.024 \\
\hline 13 & muddy-transparent & 0.753 & -0.075 \\
\hline 14 & ambiguous-distinctive & 0.692 & 0.304 \\
\hline 15 & gloomy-shiny & 0.660 & 0.126 \\
\hline 16 & delicate-harsh & -0.650 & 0.258 \\
\hline 17 & dark-bright & 0.645 & -0.078 \\
\hline 18 & old-new & 0.603 & 0.205 \\
\hline 19 & fine-coarse & -0.596 & -0.029 \\
\hline 20 & uniform-uneven & -0.488 & 0.036 \\
\hline 21 & cold-warm & 0.428 & 0.241 \\
\hline 22 & weak-powerful & 0.263 & 0.707 \\
\hline 23 & inconspicuous-conspicuous & 0.237 & 0.653 \\
\hline 24 & light-rich & 0.039 & 0.638 \\
\hline 25 & static-dynamic & 0.001 & 0.635 \\
\hline 26 & quiet-lively & 0.047 & 0.572 \\
\hline 27 & faint-deep & -0.051 & 0.562 \\
\hline 28 & showy-dull & 0.156 & -0.472 \\
\hline \multicolumn{2}{|c|}{ Eigenvalue } & 11.8 & 3.12 \\
\hline \multicolumn{2}{|c|}{ Contribution ratio (\%) } & 42.2 & 11.1 \\
\hline \multicolumn{2}{|c|}{ Cumulative contribution ratio (\%) } & 42.2 & 53.3 \\
\hline
\end{tabular}

As regards the factor loadings of SD variables for each common factor, we assume that factor loadings of an absolute magnitude greater than 0.4 are effective for the factor. According to the result of the second analysis, then, two SD items both of whose factor loadings are found ineffective have been omitted. Then, another factor analysis is carried out for the remaining 28 SD variables.

The result of the third analysis is shown in Table 1, which includes the factor loadings of the rotated values. In this table, the SD variables are divided into two groups: one that is composed of those variables whose loading of the primary common factor, referred to as Factor 1 , is 
larger than the loading of the secondary common factor, referred to as Factor 2, in absolute magnitude, and the other that is composed of those variables whose Factor 2-loading is larger than Factor 1-loading. The variables of the first group are listed in descending order of the absolute Factor 1-loadings, and then, those of the second group are listed in descending order of the absolute Factor 2-loadings. These two main common factors account for $53.3 \%$ of the total variance. Factor 1 is affected mainly by 21 variables and Factor 2 by 7 variables. Note that the SD items were actually written in Japanese in the experiment.

Figure 3 shows two dimensional plots of the pairs of factor loadings for 28 variables with the axes rotated. From this figure, we observe that the variables cluster together around the two axes that represent the respective main common factors.

\subsubsection{Interpreting common factors}

An interpretation of the meaning of each common factor extracted above is discussed below. As shown in Table 1, Factor 1 accounts for $42.2 \%$ of the total variance. Also, those SD items of a large factor loading are unlikeable-likeable, bad-good, dirty-clean and so on. These adjectives are considered to describe people's feelings and preferences. Accordingly, we designate this factor as a sensitivity factor.

Factor 2 explains $11.1 \%$ of the total variance. Those SD items of a large factor loading are weak-powerful, light-rich, static-dynamic and so on. These adjectives are considered to describe how much active, energetic or dynamic an image looks. Accordingly, we label this factor as activity. 


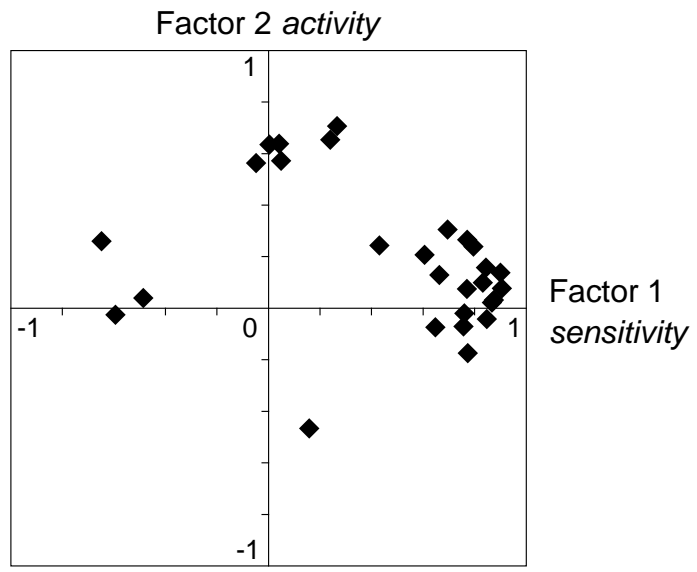

Figure 3: Two-dimensional plot of pairs of factor loadings after rotated

\subsection{Variance analysis}

By the above factor analysis, two factor scores of the respective common factors are calculated for each SD sample. Using the factor scores, we discuss the characterizing of the halftoning methods below.

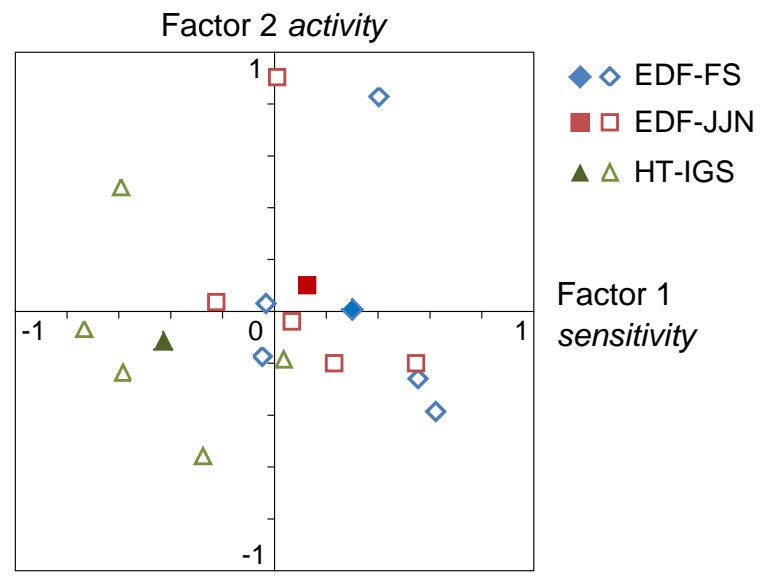

\footnotetext{
Figure 4: Two-dimensional plot of two factor scores of the respective common factors: An open marker shows the factor scores averaged for each of the five source images. A solid marker shows the average point of all the samples for each method.
}

First, for each method, we have carried out a variance analysis on the factor scores of SD samples for the five kinds of image. The result shows that both the factor scores of Factor 1 and those of Factor 2 differ among the five kinds of image at the significant level of 0.05 . Figure 4 shows two-dimensional plots of the averaged factor score of Factor 1 and that of Factor 2 for each image.

Next, another variance analysis has been carried out on the factor scores of all the SD samples for the three methods. The averaged factor score is also obtained for each method as 
depicted in preceding Figure 4. The result reveals that the factor scores of Factor 1 differ among the methods at the significant level of 0.05. Thus, Factor 1 can describe the difference in sensitivity impression among the three methods. The factor scores of Factor 2, on the contrary, show little difference among the methods.

\section{CONCLUSION}

By carrying out statistical analyses on the sample data obtained by the SD method, we have summarized visual impressions given by binary halftone images into two common factors, sensitivity and activity. The analyses have also demonstrated that EDF-FS method can produce those binary halftone images which give a stronger impression of sensitivity than other two methods, and HT-IGS method produces the least sensitivity in the black-and-white distribution. As for the activity impression, the three methods give little difference to the subjects.

The fact that one of the factors is able to differentiate among the halftoning methods validates this approach. Our future study is to investigate the impression factors in more detail so as to choose an appropriate halftoning method that can produce visual impressions as required.

\section{REFERENCES}

[1]. Ulichney, R., Digital Halftoning, MIT Press, 1993.

[2]. Venkata, N.D., Evans, B.L., and Monga, V., Color error-diffusion halftoning, IEEE Signal Pocessing Magazine, 2003, 20(4): p. 51-58.

[3]. Heise, D.R., Surveying Cultures: Discovering Shared Conceptions and Sentiments, Wiley, 2010.

[4]. Floyd, R.W. and Steinberg, L., An adaptive algorithm for spatial grayscale, Proceeding of the Society for Information Display, 17(2), 1976: p. 75-77.

[5]. Jarvis, J.F. and Judice, C.N. and Ninke, W.H., A survey of techniques for the display of continuous-tone pictures on bilevel displays, Computer Graphics and Image Processing, 5(1), 1976: p. 13-40.

[6]. Gonzalez, R.C., Woods, R.E., Digital Image Processing, Addison-Wesley, 1993.

[7]. Kimoto, T., Multi-level halftoning by IGS quantization, Journal of Signal and Information Processing, 4(4), 2013: p. 351-358.

[8]. Rosenfeld, A., Kak, A.C., Digital Picture Processing, Academic Press, 1982.

[9]. (Image files) http://rOk.us/graphics/kodak/

[10]. (Image files) http://sipi.usc.edu/database/

[11]. (Image files) http://homepages.cae.wisc.edu/ ece533/images/ 


\section{Appendices}

\section{A. DETERMINING VIEWING CONDITIONS}

\section{A.1 Experimental method}

Both for the purposes of presenting halftone images to subjects appropriately for the SD method and for the purpose of performing the presentation under the same viewing conditions, the following two variables are determined:

(a) A color of the background area surrounding a halftone image in the monitor screen

(b) A duration of time a halftone image is displayed to a viewer on the screen

The variables are evaluated by simulating the view in a preliminary experiment. The method of the experiment is described in detail below.

As regards the background color, monochrome color, that is, grayscale is here assumed to be proper because halftone images are to be displayed in black and white. Then, a halftone image is displayed in the background of an 8-bit brightness level $B$ for just $T$ seconds where $B$ takes one of five values $0,64,128,192$ and 255 and $T$ takes one of four values 2, 3, 4 and 5 . Thereby, 20 different displays are presented to every subject in a random order. For each display, a subject is asked to evaluate both the whole screen view from the viewpoint of how much easy to see it is on the ratings listed in Table A.1, and the duration of time from the viewpoint of how much suitable it is for obtaining a certain impression from the image on the ratings listed in Table A.2.

Table A.1: Ratings used to evaluate the whole screen view

\begin{tabular}{|c|l|}
\hline Value & Rating \\
\hline \hline 5 & Very easy to see \\
\hline 4 & Easy to see \\
\hline 3 & Ordinary \\
\hline 2 & Hard to see \\
\hline 1 & Very hard to see \\
\hline
\end{tabular}

Table A.2: Ratings used to evaluate the duration of displaying an image

\begin{tabular}{|c|l|}
\hline Value & Rating \\
\hline \hline 5 & Too long \\
\hline 4 & Long \\
\hline 3 & Suitable \\
\hline 2 & Short \\
\hline 1 & Too short \\
\hline
\end{tabular}

Figure A.1 shows a halftone image of $256 \times 256$ pixels produced by EDF-JJN. We use it through this preliminary experiment and display it on a 12.1" color LCD monitor of $1024 \times 768$ dots by using a $2 \times 2$ dot area for every pixel. Thus, the displayed image is $123 \mathrm{~mm}$ square centered in the $246 \mathrm{~mm} \times 185 \mathrm{~mm}$ monitor screen. The hardware settings of the monitor such as luminous intensity and contrast are fixed during the experiment. Nine subjects have taken 
part in the experiment, who are all male Japanese in their early twenties and unfamiliar with digital halftoning techniques.

\section{A.2 Analyses of experimental results}

From the above experiment, we have obtained both nine scores regarding the screen view and nine scores regarding the displaying duration for each viewing condition $(B, T)$, for the background level $B=0,64,128,192,255$ and the duration time $T=2,3,4,5$. The analyses of these results evaluate the proper values of $B$ and $T$ below.

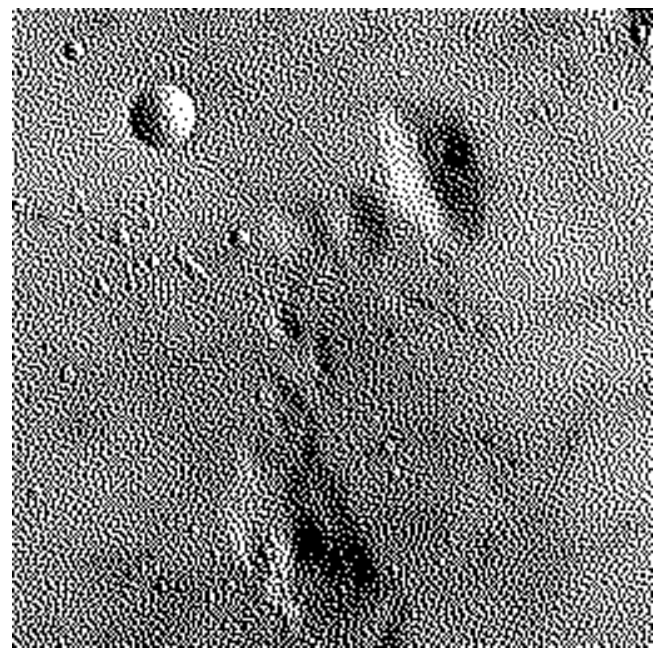

Figure A.1: The halftone image used for evaluating the viewing conditions in the preliminary experiment

\section{A.2.1. Background level}

A total of 36 scores on the ratings defined in Table A.1 have been collected for each of five values of the background level $B$. The frequency distributions of rating values in the scores are shown as a function of $B$ in Figure A.2. The figure also includes the gravity center $\mu$ of the frequencies for each $B$, which is defined as a ratio of moment by

$$
\mu=\frac{\sum_{v=1}^{5} v \cdot N(v)}{\sum_{v=1}^{5} N(v)}
$$


where $N(v)$ is the frequency of a rating value $v$ for $v=1,2, \ldots, 5$.

From Figure A.2, we observe that the total proportion of the rating values 3,4 and 5 which correspond to the rating ordinary, easy to see and very easy to see, respectively, increases as $B$ decreases to 0 , and the gravity center increases accordingly. Consequently we select the background level $B$ to be 0 .

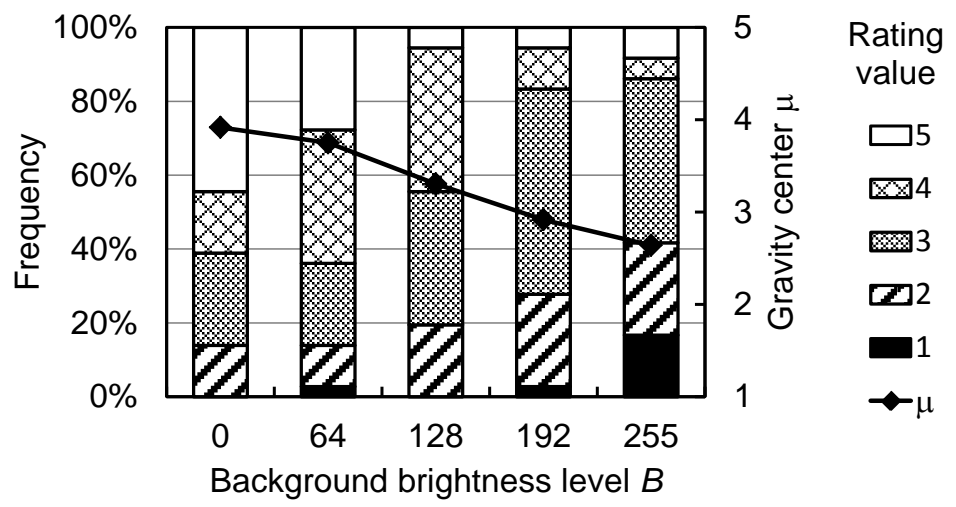

Figure A.2: Frequency distributions of the ratings on screen view for background brightness levels

\section{A.2.2. Displaying duration}

A total of 45 scores on the ratings defined in Table A.2 have been collected for each of four values of the displaying time $T$. The frequency distributions of rating values in the scores are shown for each $T$ separately in Figure A.3. Using a calculation similar to that defined in Equation (A.1), we also obtain gravity centers of the scores. Figure A.4 shows them as a function of $T$.

From Figure A.4, it is obvious that the displaying time of four seconds, $T=4$, achieves the gravity center closest to the rating value of 3 which corresponds to the rating suitable in these four experimental values of $T$, and Figure A.3(c) supports it. Consequently we select the displaying time $T$ to be four seconds. 


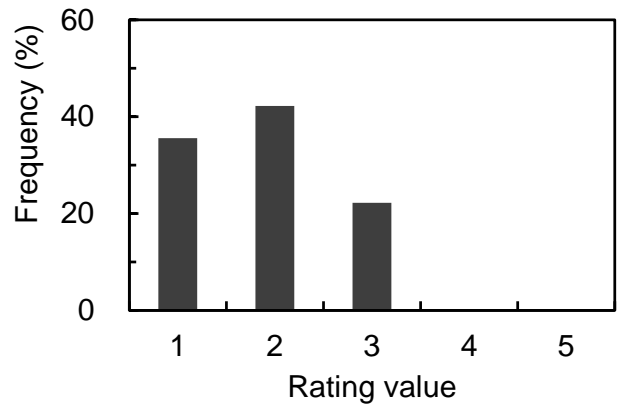

(a)

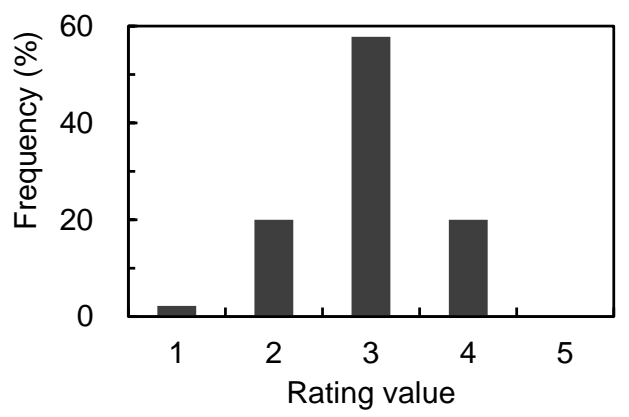

(c)

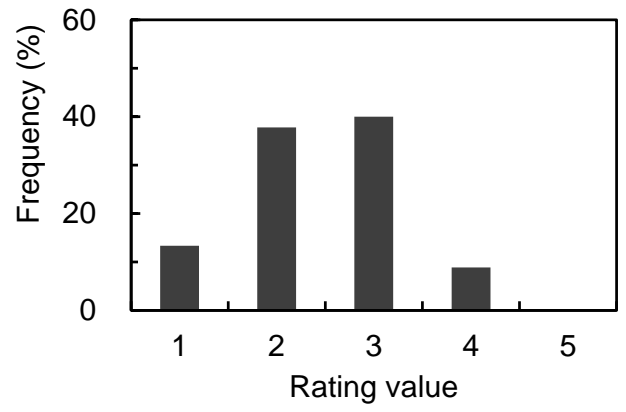

(b)

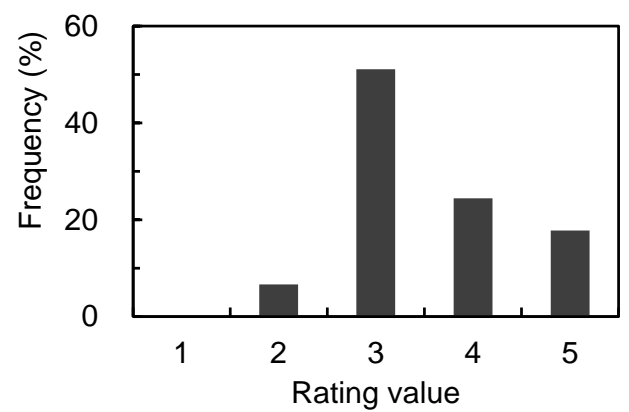

(d)

Figure A.3: Frequency distributions of the ratings on displaying duration for the time (a) $T=2$, (b) $T=3$, (c) $T=4$ and (d) $T=5$ seconds

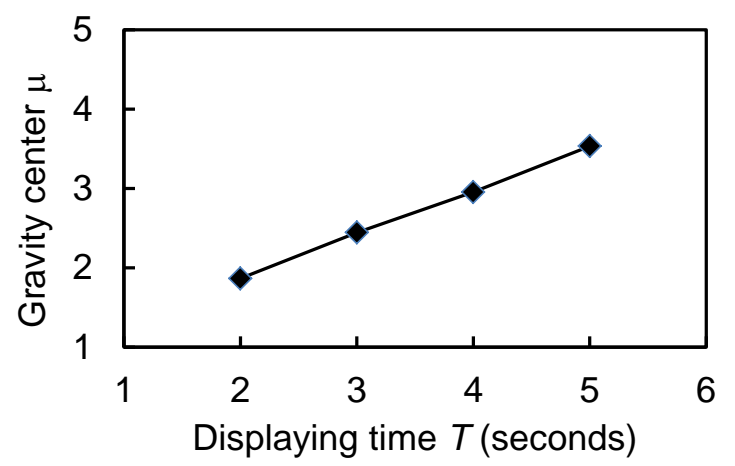

Figure A.4: Gravity center of the rating scores shown in Figure A.3

\section{B. SELECTING SD ITEMS}

\section{B.1 Experimental method}

The SD items to apply to the evaluation of halftone impressions are chosen from a source set of SD items through another preliminary experiment with subjects and test images. The source set has been composed of items of each of the following seven categories: appearance, sensibility, activity, mass and texture, conditions, sense of value and attitude and character, 
which are generally used in psychological analysis. Thus, a total of 75 SD items have been gathered in the source set. Note that these items were actually written in Japanese in the experiment.

Figure A.5 shows a set of six halftone images that was used in this preliminary experiment. Two of the images are of size $512 \times 512$ pixels, and the other images are of size $256 \times 256$ pixels. Each image was produced by one of the halftoning methods EDF-FS, EDF-JJN and IGS. The image set was printed on a single A4-size sheet of photographic paper with a full-color photo printer, which has a printing resolution of 400 dots per inch. In printing, each image was printed at the pixel resolution of either 200 pixels per inch (ppi) or 400 ppi so that any of them was shown in the same size of 2.56 inch square. Thus, the test sheet represents both a variety of halftone patterns and two kinds of halftone resolutions.

Looking at the test sheet, every subject is asked to judge for each SD item of the source set whether the item can describe an impression that the subject himself/herself gets from the images. Forty-four subjects took part in the experiment.

\section{B.2 Analysis of experimental result}

From the experimental result, we obtain the approving rate of each SD item, that is, the ratio of subjects who have approved the capability of the item of describing halftone image impressions. Figure A.6 shows the approving rates of the 75 SD items of the source set in descending order. For example, the item unclear-clear is one of the SD items of the largest approving rate of 0.7 which means that 31 of 44 subjects have approved it in this experiment. In accordance with the result, we have determined 30 SD items in the descending order of the approving rates. Table A.3 summarizes the selection of SD items, including the examples of items in each category. Again note that there might be language gaps between Japanese and English in translating in this paper.

In subjective evaluation, an SD item is to be evaluated by measuring semantic degree between the two contradictorily adjective words. We use a scale of seven gradations for the measurement; gradations 1 and 7 correspond to the respective adjective words; gradation 4 represents being intermediate, or more specifically, not impressed either way. An example of the scale is illustrated in Figure A.7. The selected SD items are to be presented to subjects with each associated scale on a questionnaire sheet in the main experiment. 


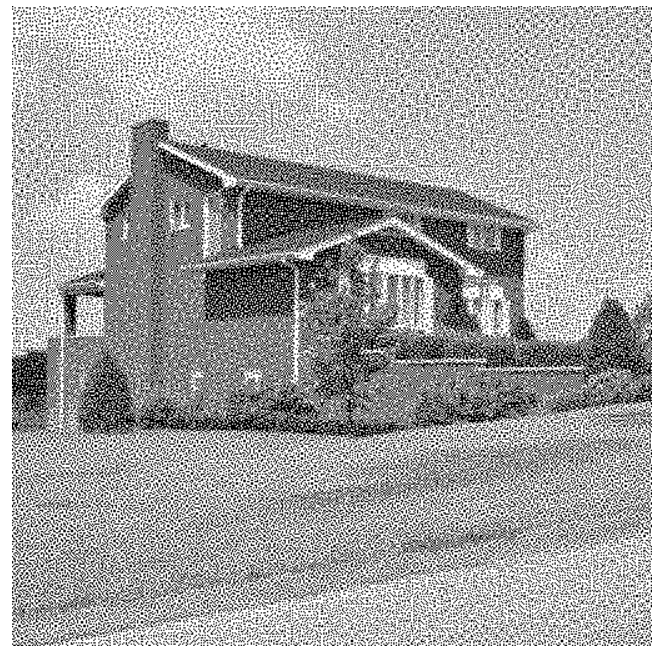

(a)

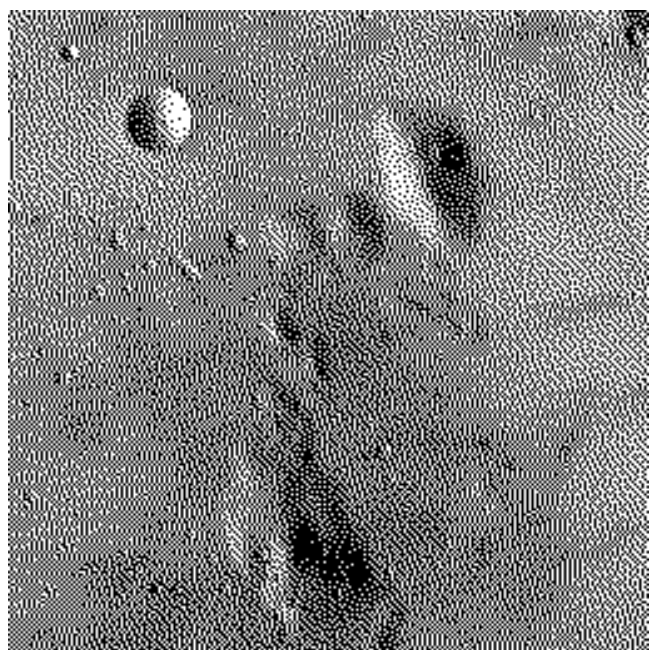

(c)

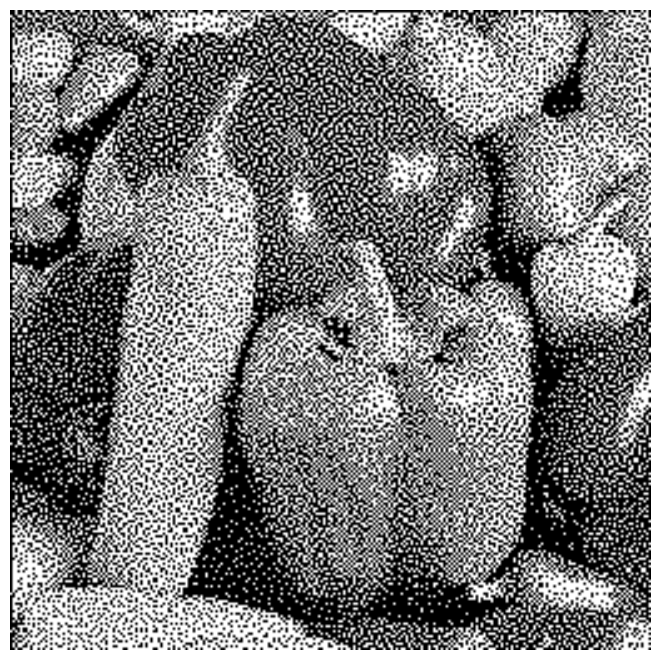

(e)

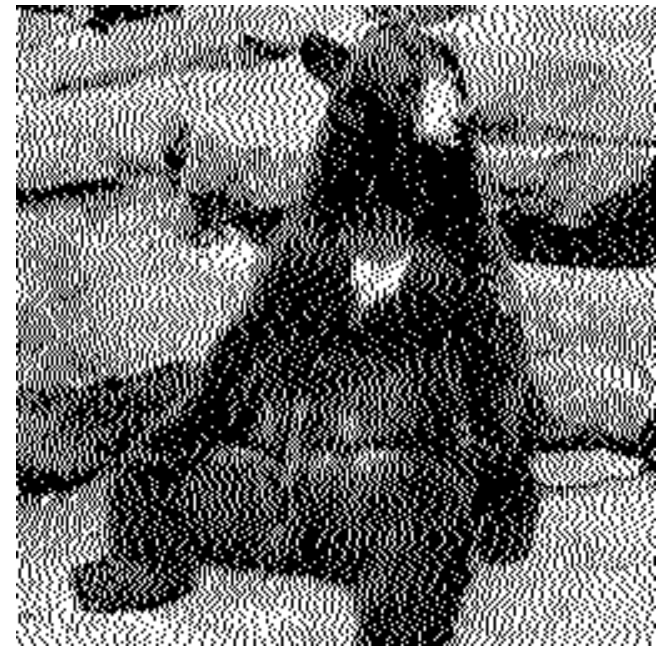

(b)

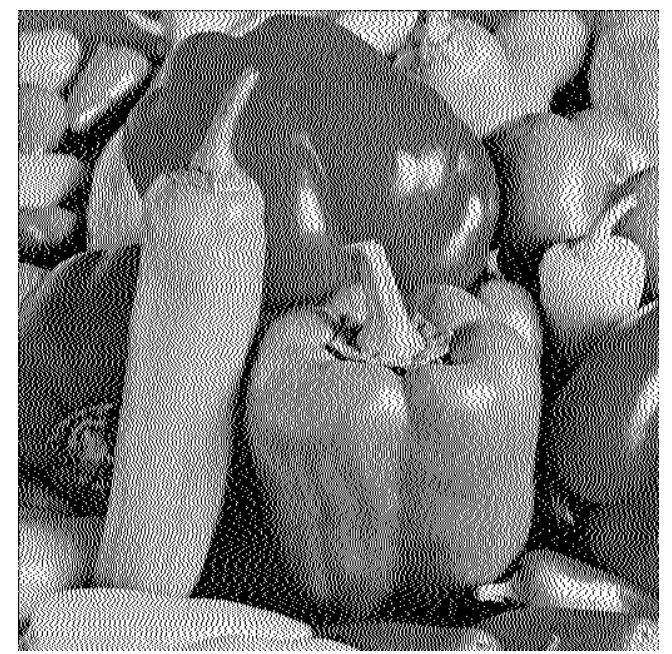

(d)

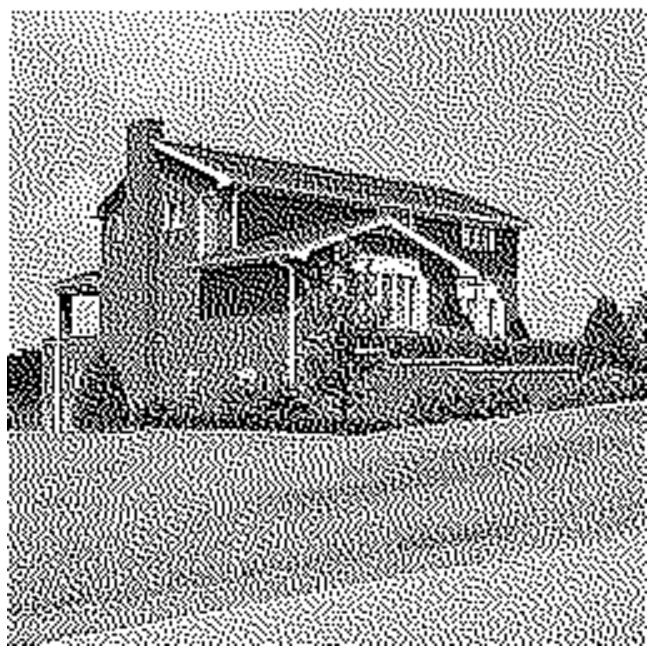

(f)

Figure A.5: Digital halftone images used in the preliminary experiment. All the images are shown here with the same size as those actually printed. The printed pixel density of (a) and (d) is 200 pixels per inch (ppi), and the others 100 ppi. The images (c) and (f) were made by the error-diffusion methods, the others by the IGS method. 


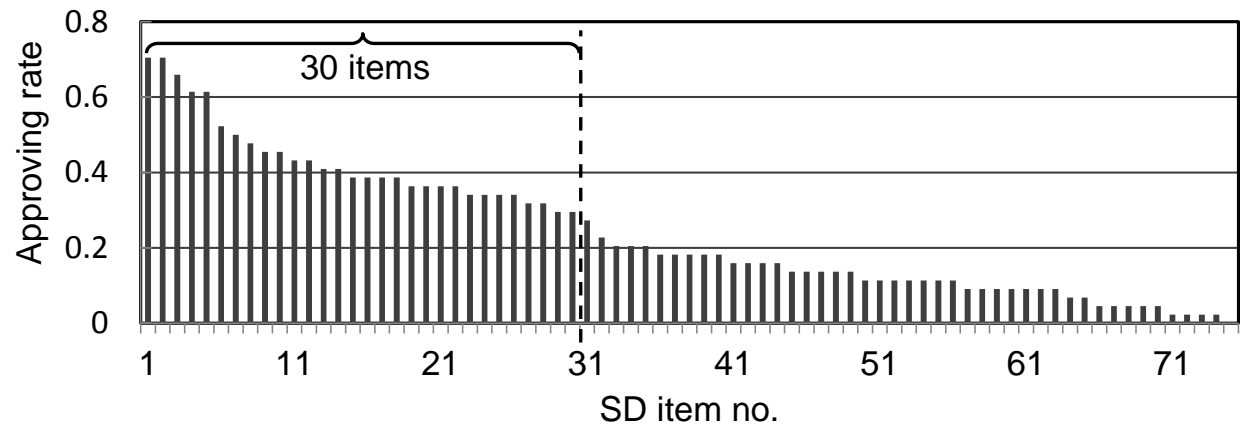

Figure A.6: Experimental result of selecting SD items

Table A.3: Selecting SD items

\begin{tabular}{|l|c|c|l|}
\hline \multicolumn{1}{|c|}{ Category } & $\begin{array}{c}\text { Number of } \\
\text { source items }\end{array}$ & $\begin{array}{c}\text { Number of } \\
\text { selected items }\end{array}$ & \multicolumn{1}{|c|}{ Examples } \\
\hline \hline Appearance & 11 & 9 & $\begin{array}{l}\text { dark-bright } \\
\text { ambiguous-distinctive }\end{array}$ \\
\hline Sensibility & 7 & 5 & $\begin{array}{l}\text { ugly-beautiful } \\
\text { cold-warm }\end{array}$ \\
\hline Activity & 6 & 3 & $\begin{array}{l}\text { static-dynamic } \\
\text { weak-powerful }\end{array}$ \\
\hline Mass and texture & 7 & 4 & $\begin{array}{l}\text { fine-coarse } \\
\text { gloomy-shiny }\end{array}$ \\
\hline Conditions & 14 & 4 & $\begin{array}{l}\text { unnatural-natural } \\
\text { old-new }\end{array}$ \\
\hline Sense of value & 4 & 1 & $\begin{array}{l}\text { low quality-high quality } \\
\text { inexpensive-expensive }\end{array}$ \\
\hline Attitude and character & 26 & 4 & $\begin{array}{l}\text { delicate-harsh } \\
\text { unfriendly-friendly }\end{array}$ \\
\hline \hline Total & 75 & 30 & - \\
\hline
\end{tabular}

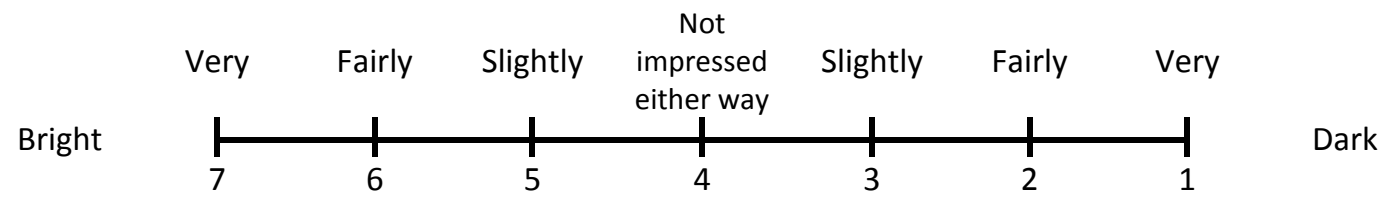

Figure A.7: Example of scale for replying to an SD item dark-bright 